\title{
Electrochemical study of hydrogen diffusion in yttrium hydride switchable mirrors
}

\author{
M. Di Vece*, J.J. Kelly \\ Physics and Chemistry of Condensed Matter, Debye Institute, Utrecht University, P.O. Box 80000,3508 TA Utrecht, The Netherlands
}

Received 1 June 2002; accepted 25 October 2002

\begin{abstract}
Potential-step experiments on yttrium thin films show exponential decay of the current as a function of time. A diffusion model for a thin-layer system relates the time constants to effective diffusion coefficients for hydrogen in the di- and trihydride concentration range. The diffusion coefficient is found to be concentration-dependent. From the temperature dependence of the diffusion coefficients and the optical switching time, activation energies are calculated. The good agreement with literature values supports the model of diffusionlimited kinetics for the optical switching.
\end{abstract}

(C) 2002 Elsevier B.V. All rights reserved.

Keywords: Thin films; Electrochemical reactions; Dielectric response

\section{Introduction}

The switchable mirror, a new class of electrochromic material discovered in 1996 [1], is currently the subject of much fundamental and applied research [2]. Thin metal hydride films allow a controlled change in reflectance, absorbance and transmittance of light. A reversible metal to insulator transition is responsible for the optical changes when the metal is switched between the dihydride and the trihydride states. The metal state is opaque, while the insulator is transparent.

Hydrogen diffusion may limit the switching time, and is therefore of considerable importance. Mobility coefficients for lateral transport of hydrogen in yttrium thin films have been determined from optical measurements during loading from the gas phase [3]. Van der Molen et al. [4] compared hydrogen diffusion perpendicular to the matrix sample surface with lateral transport (in gas phase experiments). It was shown that the switching time and the morphology of the film depend strongly on the film thickness. Since it is known that hydrogen diffusion coefficients can depend strongly on film thickness [5], the relation between the switching time and diffusion coefficient remains a matter of interest.

Electrochemical loading has the advantage that the

*Corresponding author.

E-mail address: m.divece@phys.uu.nl (M. Di Vece). concentration of hydrogen in the film, the hydrogen/metal atomic ratio, $x$, can be controlled and monitored accurately on the basis of the charge transferred in the experiment [6]. The current is directly proportional to the hydrogen flux at the surface of the film. From the current and transmission transients, information can be obtained about the switching kinetics. In this report we investigate switching by potential step experiments in which both the current and transmission are followed as a function of time.

\section{Experimental}

The active layers were nominally $200 \mathrm{~nm}$ thick, polycrystalline Y films top-coated with a thin palladium layer (nominal thickness $10 \mathrm{~nm}$ ) which acts as a catalyst for hydrogenation and protects the film from oxidation. Between the active layer and the glass substrate a 15 -nm gold layer with a $2.5-\mathrm{nm}$ chromium adhesion layer was deposited for electrical contact. The active film covered only part of the gold surface, to allow a back contact to the film via the gold. The layers were deposited by evaporation at $10^{-9}$ mbar base pressure. A platinum lead was fixed with silver glue (2400 Circuit Works Conductive Epoxy, Agar Scientific) on the free gold, and both the lead and the gold were protected from the solution by Apiezon. The active surface area was $1.2 \mathrm{~cm}^{2}$.

A two-compartment electrochemical glass cell was used 
for the measurements. The working electrode was positioned together with a $\mathrm{Hg} / \mathrm{HgO}$ reference electrode in one compartment and a platinum counter electrode was placed in the other compartment. All potentials are given with respect to $\mathrm{Hg} / \mathrm{HgO}$. Argon gas was bubbled through the solution in both chambers. The glass cell had windows on both sides through which we could illuminate the sample and measure transmission with a diode laser (Vector, $670 \mathrm{~nm}$ ) and a standard photodiode. All electrochemical experiments were carried out in a $1 \mathrm{M} \mathrm{KOH}$ solution at room temperature. A potentiostat/galvanostat (EG\&G Princeton Applied Research (PAR) 273A) was computer-controlled by in-house developed programs (LabView).

\section{Results and discussion}

The yttrium thin films were galvanostatically loaded and switched several times to exclude virgin effects. Because the hydrogen cannot be removed from the film for concentration ratios lower than $x=1.8$, the experiments were limited to the reversible hydrogen loading range, i.e. to the dihydride-trihydride transition [1]. Current and transmission transients were recorded simultaneously after a step from $0.2 \mathrm{~V}$ to $-0.95 \mathrm{~V}$. Two different time domains ( $t \leq 100 \mathrm{~ms}$ and $1 \leq t \leq 60 \mathrm{~s}$ ) were investigated in separate experiments. Initial current densities of $150 \mathrm{~mA} / \mathrm{cm}^{2}$ were observed and attributed to hydrogen absorption by the palladium caplayer. In Fig. 1 a typical current transient in the long time domain is shown. In the first seconds, a fast decay in the current is clear. This is followed by a much slower decay which is accompanied by the main optical change, as can be seen from the transmission transient. The slight initial decrease in the transmission is caused by an optical window [6,7] at around $x=2$, which is the starting point for the transients. Van Gogh et al. [8] explain this feature by a screening of the plasma energy and a low interband absorption coefficient, leading to lower absorption. The current transients of Fig. 1 can be fitted with a double exponential function, using five parameters: preexponential terms $A_{1}$ and $A_{2}$, time constants $t_{1}$ and $t_{2}$ and $y_{0}$, a small steady-state current.

$I(t)=y_{0}+A_{1} \exp \left[-\frac{t}{t_{1}}\right]+A_{2} \exp \left[-\frac{t}{t_{2}}\right]$

An example of a fit is shown by the dashed line in Fig. 1. An exponential current transient following a potential step, is reminiscent of results reported on an analogous system, the intercalation of lithium in thin films [9]. Wen et al. [9] explained this dependence by diffusion, using a model consisting of two parallel plates; the back side is impermeable to the diffusing species while the concentration at the electrolyte side is fixed at a constant value. Before the potential step, the concentration in the film is uniform and constant. Solving Fick's diffusion equations [10] and taking first-order terms, Wen et al. have shown that the current is given by [9]

$I(t)=\frac{2 F S D \Delta C}{l} \cdot \exp \left[\frac{-D \pi^{2} t}{4 l^{2}}\right] t>l^{2} / D$

where $D$ is the diffusion coefficient, $S$ the active surface area, $l$ the film thickness, $F$ the Faraday constant and $\Delta C$ the concentration difference, calculated from the total charge transferred in the reaction. This allows one to determine effective diffusion coefficients from the time dependence of the current. Assuming this model to be valid for our system, we can calculate effective diffusion coefficients for hydrogen transport. A fit of the exponential decay of the current provides an average diffusion coefficient of the reversibly loaded hydrogen. Inspection of the approximation leading to Eq. (2) shows that for $t \geq 0.2 \cdot l^{2} / D$ an error of $\leq 10 \%$ will be made. The

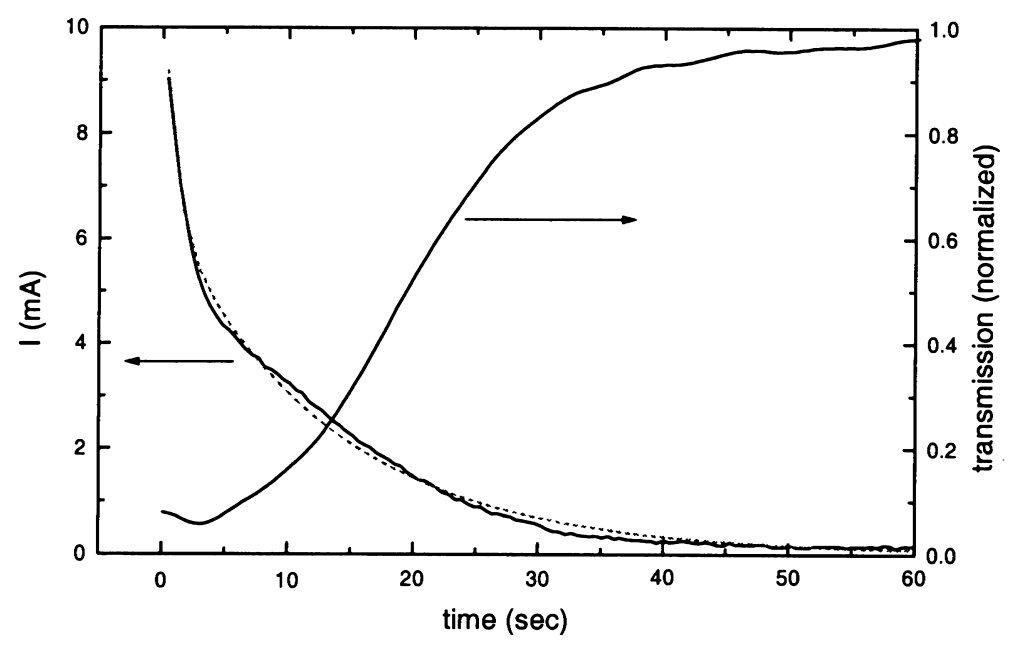

Fig. 1. Transient of current (solid line) with fit (dashed-dotted line) and of transmission (dashed) of an yttrium thin film after a potential step from $0.2 \mathrm{~V}$ to $-0.95 \mathrm{~V}$. 


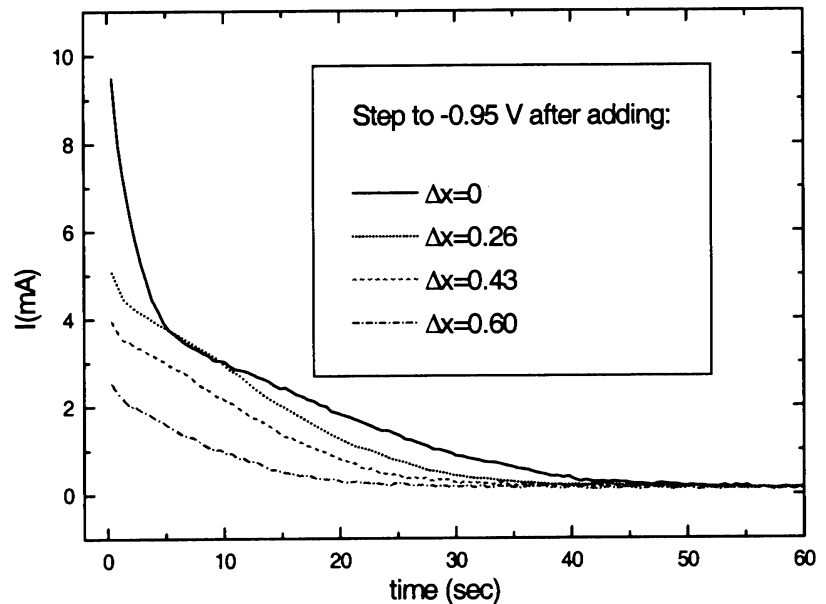

(A)

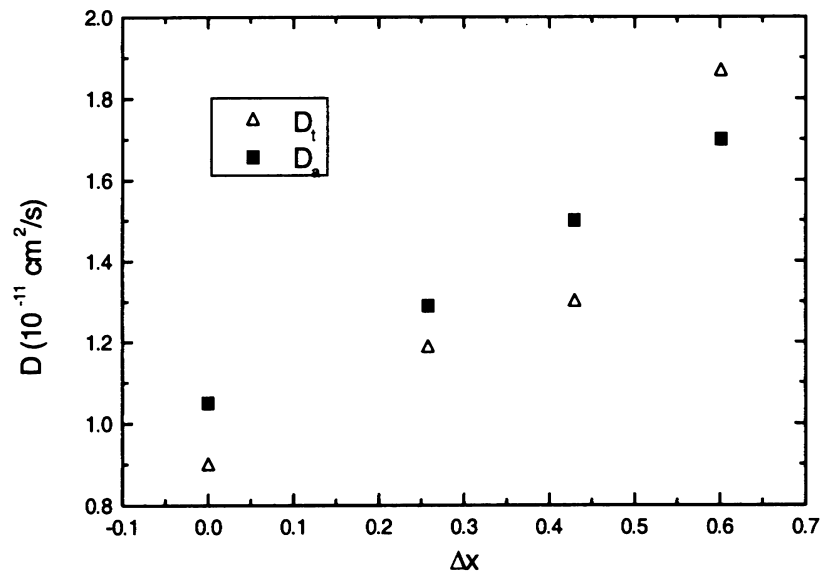

(B)

Fig. 2. (A) Current transients after addition of different amounts of hydrogen $\Delta x$. (B) Diffusion coefficients calculated from the current transients with Eq. (2). $D_{\mathrm{t}}$ was obtained from the exponent and $D_{\mathrm{a}}$ from the preexponential factor from the fit with Eq. (1). remarkable agreement between the diffusion coefficients determined from the prefactor and the exponent, as discussed later, confirms the validity of this model.

In Fig. 2(A), current transients are shown for different starting concentration ratios $x$. This was accomplished by introducing additional charge, i.e. $\Delta x$, before the potential step. The fast exponential decay, clearly visible in the transient without extra hydrogen $(\Delta x=0)$, is almost absent for $\Delta x=0.26$. For larger $\Delta x$, the transient can be fitted with a single exponential decay function. The presence of a double decay process can be understood in the context of the different phases through which loading occurs. The first decay in the transient very likely corresponds to the filling of the $\beta$-phase with a higher diffusion coefficient. The slow decay is then related to the transformation in the $\beta-\gamma$ phase. According to the phase diagram of the yttrium hydride thin film [1], the $\beta$ phase corresponds to $\Delta x=0.24$, which is in good agreement with our result for the fast decay.

The diffusion coefficients calculated with Eqs. (1) and (2) for the slow decay, are shown in Fig. 2(B) as a function of $\Delta x$. The remarkably good agreement between the diffusion coefficients calculated from the prefactor and the exponent, support the validity of the diffusion model. An increase in the diffusion coefficient as a function of concentration is apparent. This is maybe due to a change in electronic structure at higher concentrations of hydrogen, giving rise to a lower diffusion barrier [11].

The temperature dependence of the diffusion coefficients determined from the preexponential and exponential factors is displayed in Fig. 3, for the fast and slow decay. The subindices $t$ and $A$ refer to the exponential and preexponential fit parameters, respectively of Eq. (1). The activation energies calculated from the slopes are 0.39 and $0.34 \mathrm{eV}$, respectively. Assuming diffusion-controlled kinet-

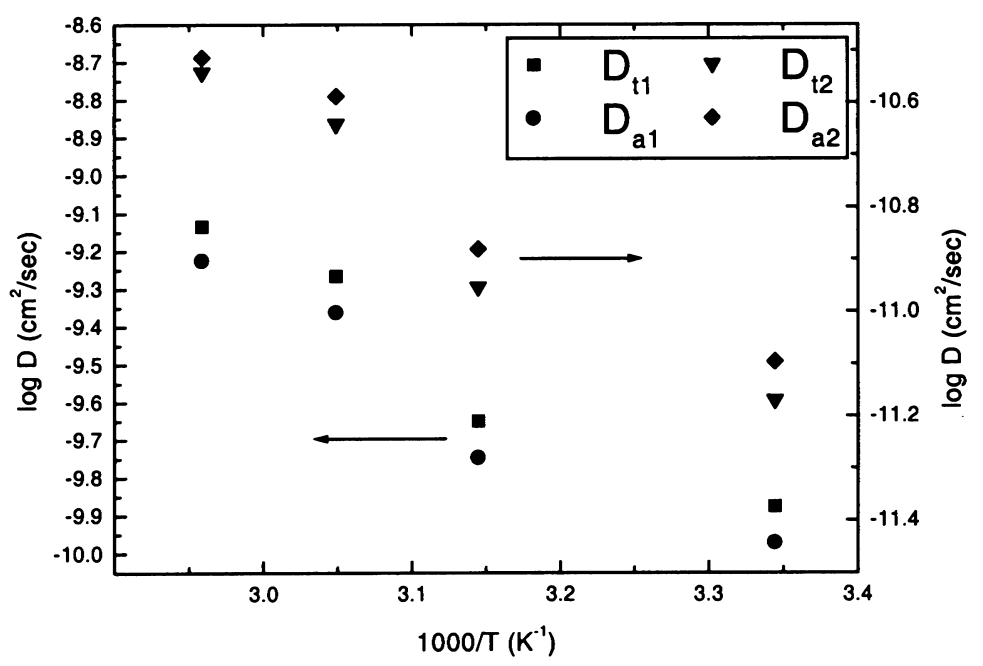

Fig. 3. Temperature dependence of the diffusion coefficients determined from the exponential current decays. $D_{\mathrm{t}}$ was obtained from the exponential and $D_{\mathrm{a}}$ from the preexponential factor from the fit with Eq. (1). 


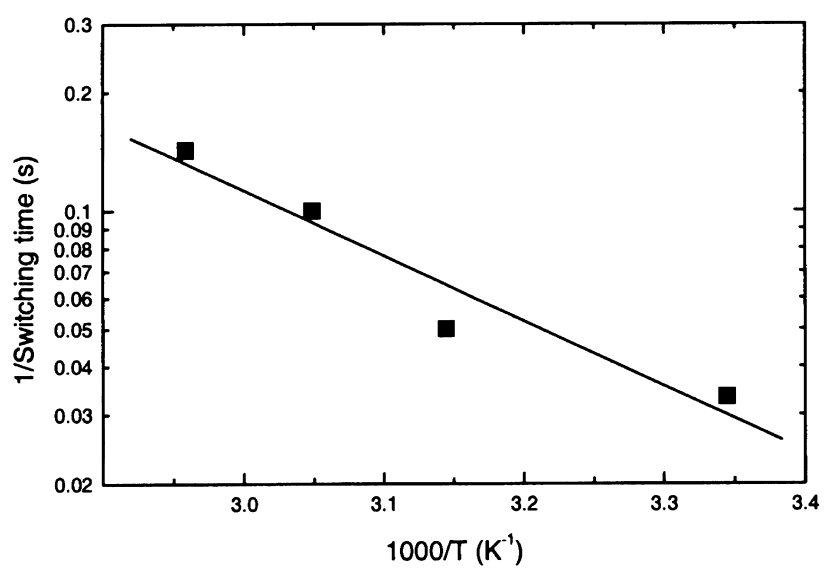

Fig. 4. Temperature dependence of the reciprocal switching time shows Arrhenius behaviour.

ics for the optical switching, we can relate the switching time $t_{\text {switch }}$ to $D\left(t_{\text {switch }} \approx l^{2} / D\right)$, in which $t_{\text {switch }}$ is defined as the maximum of the time derivative of the transmission. In Fig. 4, the reciprocal switching time is plotted as a function of $T^{-1}$. From the slope, we calculate an activation energy of $0.33 \mathrm{eV}$, very close to the value corresponding to the slow current decay. Since the optical change lies within the slow current decay domain, the good agreement between both activation energies is not surprising. For NMR studies, Majer et al. [12] report an activation energy of $0.38 \mathrm{eV}$ for yttrium powder with $x=2.03$, in close agreement with our value. Lateral gas-phase loading in yttrium thin films [3] provides an activation energy of 0.37 $\mathrm{eV}$ for the $\beta-\gamma$ transition. In this lateral experiment, the $\beta-\gamma$ phase boundary is visible as transmission discontinuity, with a $t^{1 / 2}$ dependence. The extremely small dimension of $l$ for loading, normal to the surface, provides too little time for the formation of a phase boundary in our experiments.

\section{Conclusions}

A simple thin-layer diffusion model provides effective diffusion coefficients for hydrogen in yttrium, from the exponential decay of the current after application of a potential step. A concentration dependence of the diffusion coefficient has been found. Experiments at different temperatures show an Arrhenius dependence of both the diffusion coefficients and the switching time. The corresponding activation energies agree well with literature values.

\section{Acknowledgements}

The authors would like to thank their colleagues at the Vrije Universiteit Amsterdam: R. Griessen, A. Remhof and I.A.M.E. Giebels for stimulating discussions, N. Koeman for preparation of the samples. The work described here was supported by the Council for Chemical Sciences (CW), with financial aid from the Netherlands Organization for Scientific Research (NWO) and the Netherlands Foundation for Technical Research (STW).

\section{References}

[1] J.N. Huiberts, R. Griessen, J.H. Rector, R.J. Wijngaarden, J.P. Dekker, D.G. de Groot, N.J. Koeman, Nature 380 (1996) 231.

[2] P. van der Sluis, V.M.M. Mercier, Electrochim. Acta 46 (2001) 2167.

[3] F.J.A. den Broeder, S.J. van der Molen, M. Kremers, J.N. Huiberts, D.G. Nagengast, A.T.M. van Gogh, W.H. Huisman, N.J. Koeman, B. Dam, J.H. Rector, S. Plota, M. Haaksma, R.M.N. Hanzen, R.M. Jungblut, P.A. Duine, R. Griessen, Nature 394 (1998) 656.

[4] S.J. van der Molen, J.W.J. Kerssemakers, J.H. Rector, N.J. Koeman, B. Dam, R. Griessen, J. Appl. Phys. 86 (1999) 6107.

[5] Y. Li, Y.T. Cheng, Int. J. Hydrogen Energy 21 (1996) 281.

[6] E.S. Kooij, A.T.M. van Gogh, R. Griessen, J. Electrochem. Soc. 146 (1999) 2990.

[7] P.H.L. Notten, M. Kremers, R. Griessen, J. Electrochem. Soc. 143 (1996) 3348.

[8] A.T.M. van Gogh, D.G. Nagengast, E.S. Kooij, N.J. Koeman, J.H. Rector, R. Griessen, Phys. Rev. B 63 (2001) 195105.

[9] C.J. Wen, B.A. Boukamp, R.A. Huggins, J. Electrochem. Soc. 126 (1979) 2258.

[10] J. Crank, The Mathematics of Diffusion, 2nd Edition, Oxford University Press, 1999.

[11] G. Majer, U. Kaess, R.G. Barnes, Phys. Rev. Lett. 83 (1999) 340.

[12] G. Majer, J. Gottwald, D.T. Peterson, R.G. Barnes, J. Alloys Comp. 330-332 (2002) 438. 